\title{
Universal scaling for the spin-electricity conversion on surface states of topological insulators
}

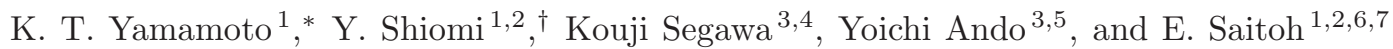 \\ 1 Institute for Materials Research, Tohoku University, Sendai 980-8577, Japan \\ 2 Spin Quantum Rectification Project, ERATO, Japan Science and Technology Agency, Aoba-ku, Sendai 980-8577, Japan \\ ${ }^{3}$ Institute of Scientific and Industrial Research, Osaka University, Ibaraki, Osaka 567-0047, Japan \\ 4 Department of Physics, Kyoto Sangyo University, Kyoto 603-8555, Japan. \\ 5 Institute of Physics II, University of Cologne, D-50937 Cologne, Germany \\ ${ }^{6}$ WPI Advanced Institute for Materials Research, \\ Tohoku University, Sendai 980-85r7, Japan and \\ 7 Advanced Science Research Center, Japan Atomic Energy Agency, Tokai 319-1195, Japan
}

(Dated: June 27, 2021)

\begin{abstract}
We have investigated spin-electricity conversion on surface states of bulk-insulating topological insulator (TI) materials using a spin pumping technique. The sample structure is $\mathrm{Ni-Fe}|\mathrm{Cu}| \mathrm{TI}$ trilayers, in which magnetic proximity effects on the TI surfaces are negligibly small owing to the inserted $\mathrm{Cu}$ layer. Voltage signals produced by the spin-electricity conversion are clearly observed, and enhanced with decreasing temperature in line with the dominated surface transport at lower temperatures. The efficiency of the spin-electricity conversion is greater for TI samples with higher resistivity of bulk states and longer mean free path of surface states, consistent with the surface spin-electricity conversion.
\end{abstract}

Injection and detection of non-equilibrium spins are key techniques in the field of spintronics [1]. A powerful method to inject spins is spin pumping. Spin pumping enables dynamical spin injection from a ferromagnet into an adjacent non-magnetic metal, which is induced by coherent precession of magnetization at ferromagnetic resonance (FMR) 2]. In spin pumping experiments, bilayers comprising ferromagnetic $\mathrm{Ni}_{81} \mathrm{Fe}_{19}$ (permalloy, Py) and nonmagnetic $\mathrm{Pt}$ have been studied as a typical system [2 6]. Though Pt has been widely used for spin detection owing to its strong spin-orbit interaction, search of more efficient spin detectors is one of the urgent issues in the spintronics field $[7,[8]$.

A topological insulator (TI) is a promising material for spintronics application because of its potential for highly efficient spin-electricity conversion [9, 10]. Topological insulators are a state of quantum matter 1113], in which the surface is metallic, while the interior is insulating. Spin-electricity conversion on TI materials has recently been investigated using spin pumping for bulk-metallic samples $\left(\mathrm{Bi}_{2} \mathrm{Se}_{3}\right)$ [14 16 ] and also for bulk-insulating ones [17]. In a previous report [17], some authors of the present paper demonstrated the spinelectricity conversion induced by spin pumping into surface states of TIs, $\mathrm{Bi}_{1.5} \mathrm{Sb}_{0.5} \mathrm{Te}_{1.7} \mathrm{Se}_{1.3}$ (BSTS) 18, 19] and Sn-doped $\mathrm{Bi}_{2} \mathrm{Te}_{2} \mathrm{Se}$ (SnBTS) 20] in contact with Py. Since millimeter-thick TI samples were used in [17], the inverse spin Hall signal from bulk carriers are neglected and the observed spin-electricity conversion signal was safely ascribed to a surface contribution. The sign of

${ }^{*}$ K. T. Yamamoto and Y. Shiomi contributed equally to this work. ${ }^{\dagger}$ Corresponding author; Electronic address: shiomi@imr.tohoku.ac.jp the generated electric signals is consistent with the spinelectricity conversion on the topological surface state, whereas the opposite sign is expected for co-existing Rashba surface states [21]. On the surface states of TIs, since spin direction and electron-flow direction have oneto-one correspondence (the spin-momentum locking), injected spins are converted into electric currents along an in-plane direction on the surface, when the bulk state is sufficiently insulating. Although highly efficient spinelectricity conversion has been reported for bulk-metallic TIs [9, 10, 16], the reported value for bulk-insulating BSTS is $\sim 0.01 \%$ [17, 22].

Though the spin-electricity conversion on the topological surface states was demonstrated using a spin pumping technique 17], it remains unclear how the produced electric signal is related to surface transport properties of TI materials. In this manuscript, we study spin-electricity conversion induced by spin pumping for several SnBTS samples whose bulk insulating properties are ideal for its detailed study. By inserting a thin $\mathrm{Cu}$ layer between Py and TI layers, magnetic proximity effects to TI surfaces are negligible. The efficiency for the observed spinelectricity conversion are found to be greater for TI materials with higher bulk resistivity and longer surface mean path. This result shows that the spin-electricity conversion takes places at the surface state and also that its efficiency strongly depends on surface transport properties.

We used SnBTS to investigate the spin-electricity conversion effect on topological surface states. The single crystals of $0.5 \% \mathrm{Sn}$-doped $\mathrm{Bi}_{2} \mathrm{Te}_{2} \mathrm{Se}$ were synthesized by a Bridgman method [20]. Since the bulk state of SnBTS is more insulating than that of BSTS [20, 23], SnBTS has been considered as an ideal system for transport study of Dirac surface states 23]. Three SnBTS samples (SnBTS1 whose size is $3.3 \times 1.2 \times 0.4 \mathrm{~mm}^{3}$, SnBTS2 $3.3 \times 2.2 \times 0.3$ 


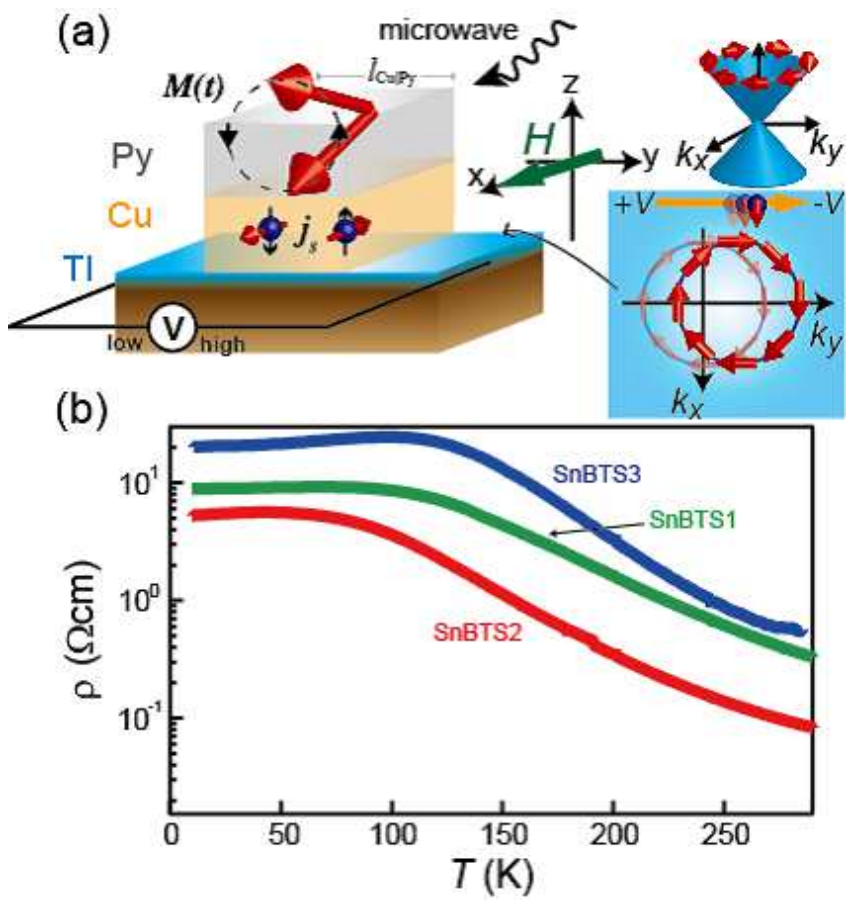

FIG. 1: (Color online.) (a) Experimental setup of spinelectricity conversion induced by spin pumping into topological insulators (TIs). "high" and "low" indicate input codes of the nanovoltmeter (Keithley 2182A). On the topological surface state, spin polarization produces spin-electricity conversion voltage in the Hall direction. The origin of this electric signal is a shift of the surface Fermi surface induced by spin injection. (b) Temperature ( $T$ ) dependence of the in-plane resistivity $(\rho)$ for three $\mathrm{Sn}$-doped $\mathrm{Bi}_{2} \mathrm{Te}_{2}$ Se samples (SnBTS1, SnBTS2, and SnBTS3).

$\mathrm{mm}^{3}$, and SnBTS3 $3.0 \times 1.5 \times 0.7 \mathrm{~mm}^{3}$ ) were used in this study. These samples were cut from one boule of SnBTS crystals.

The experimental setup of the spin-pumping measurement is almost the same as that in our previous report [17]. Schematic illustrations of the sample and experimental setup are shown in Fig. 1(a). A 5-nm-thick $\mathrm{Cu}$ film and 25-nm-thick Py film were evaporated on the middle part of a cleaved surface of TI samples in a high vacuum. The roughness of TI surfaces measured by atomic force microscopy is about $1 \mathrm{~nm}$. The length of $\mathrm{Cu}$ and Py films $\left(l_{\mathrm{Cu} \mid \mathrm{Py}}\right)$ is $0.5 \mathrm{~mm}$. In the spin-pumping measurement, magnetization dynamics in Py was excited by a microwave magnetic field on a coplanar-type waveguide in an inplane static magnetic field $(H)$. We used a commercial network analyzer as a microwave source. Microwave frequency was kept at $5 \mathrm{GHz}$ and the power of incident microwave was amplified 1000 times by a commercial microwave amplifier. While sweeping the external magnetic field, FMR spectrum in Py and electromotive force arising between the ends of TI samples were recorded simultaneously using the network analyzer and a nanovoltmeter, respectively. The measurements were
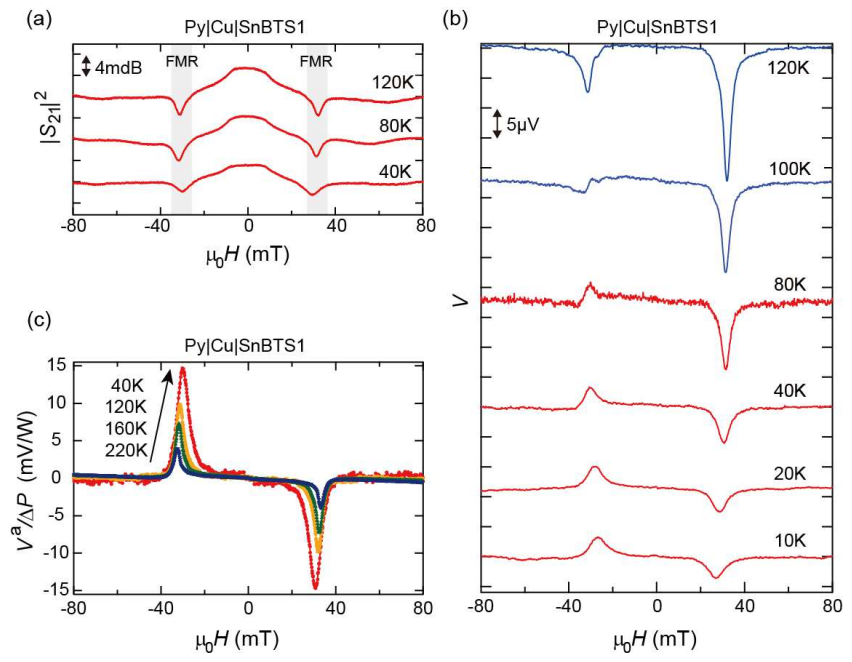

FIG. 2: (Color online.) (a) Magnetic field $(H)$ dependence of microwave transmittance, $\left|S_{21}\right|^{2}$, for $\mathrm{Py}|\mathrm{Cu}| \mathrm{SnBTS} 1$ at several temperatures. The data is shifted vertically just for clarity. (b) Magnetic field $(H)$ dependence of voltage signal $(V)$ arising around FMR magnetic-fields of Py for Py|Cu|SnBTS1 at various temperatures. The data is shifted vertically just for clarity. (c) $H$ dependence of the antisymmetric part of $V$ $\left(V^{a}\right)$ normalized by the resonance absorption power $(\Delta P)$ for $\mathrm{Py}|\mathrm{Cu}| \mathrm{SnBTS} 1$ at some temperatures.

conducted at low temperatures down to $10 \mathrm{~K}$ in a probe station.

Figure 1(b) shows temperature $(T)$ dependence of resistivity, $\rho$, for the TI samples. $\rho$ at room temperature is $0.05 \Omega \mathrm{cm}-1 \Omega \mathrm{cm}$ and increases with decreasing $T$, which indicates that the bulk carriers are compensated in all the TI samples. Using the activation law above 200 $\mathrm{K}$, values of energy gap are estimated to be $80 \mathrm{meV}-100$ $\mathrm{meV}$, similar to a reported value $(65 \mathrm{meV})$ [20]. At low temperatures below $\sim 100 \mathrm{~K}$, the resistivity begins to decrease, which is unusual for traditional semiconductors. Metallic surface conduction is dominant in the low- $T$ region.

We performed spin pumping experiments for the TI samples attached with $\mathrm{Cu}$ and Py films. Figure 2(a) shows the magnetic field $(H)$ dependence of microwave transmittance, $\left|S_{21}\right|^{2}$, for $\mathrm{Py}|\mathrm{Cu}| \mathrm{SnBTS} 1$ at several temperatures. Clear dips which correspond to ferromagnetic resonance (FMR) in Py were observed at each temperature around $\pm 30 \mathrm{mT}$. The magnitude of resonance absorption slightly decreases with decreasing $T$ because of an increase in the microwave loss in our microwave circuit.

Figure 2(b) shows the magnetic field $(H)$ dependence of voltage signals arising at FMR magnetic fields for $\mathrm{Py}|\mathrm{Cu}| \mathrm{SnBTS} 1$ at various temperatures. At $120 \mathrm{~K}$, the voltage peaks are clearly observed at FMR magnetic fields $\left( \pm H_{F M R}\right)$ of Py. The sign of the voltage peaks is negative both at $+H_{F M R}$ and $-H_{F M R}$, and their magnitudes are different. At high temperatures, the See- 


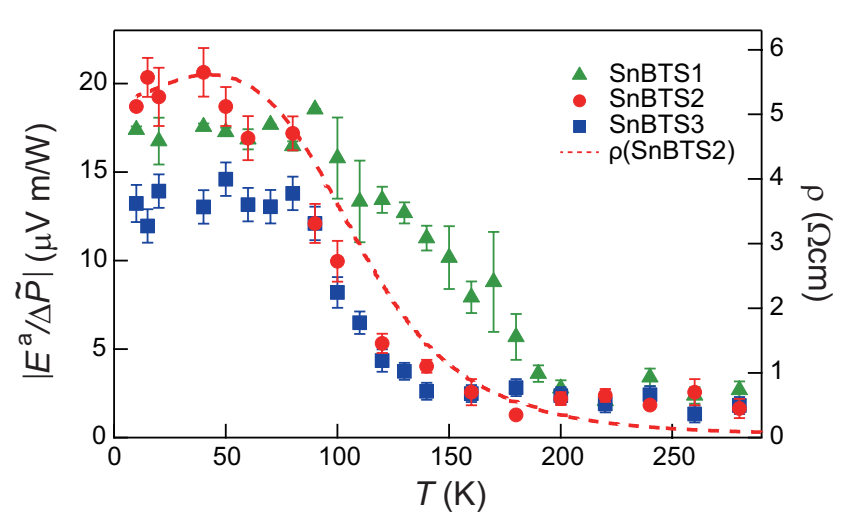

FIG. 3: (Color online.) Temperature $(T)$ dependence of the antisymmetric part of the electric field, $E^{a}\left(=V^{a} / l_{\mathrm{Cu} \mid \mathrm{Py}}\right)$, divided by the resonance absorption power per unit area $(\Delta \tilde{P})$ for the $\mathrm{Py}|\mathrm{Cu}| \mathrm{SnBTS}$ samples. The absolute values of $E^{a} / \Delta \tilde{P}$ are plotted. The $T$ dependence of resistivity $(\rho)$ for SnBTS2 is shown for comparison (dotted curve).

beck effect of bulk carriers independent of magnetic fields dominates the peak signals [17]. An origin of this Seebeck voltage is an inplane temperature gradient due to the inevitable heating effects at FMR [17]. As temperature decreases, the magnitude of the Seebeck voltage decreases since the excitation of bulk carriers is suppressed at lower temperatures. Below $80 \mathrm{~K}$, the sign reversal between $+H_{F M R}$ and $-H_{F M R}$ is observed as shown in Fig. 2(b), indicating that the spin-electricity conversion signal is dominant. The peak sign at $+H_{F M R}$ is negative, consistent with that reported in [17].

The magnitude of the spin-electricity conversion voltage increases with decreasing temperature, consistent with the dominant surface conduction at lower temperatures. Figure 2(c) shows the $H$ dependence of the antisymmetric part of the voltage peak $\left|V^{a}\right| \equiv \mid V(H)-$ $V(-H) \mid / 2$ divided by resonance absorption power $\Delta P$ for $\mathrm{Py}|\mathrm{Cu}| \mathrm{SnBTS1}$. Here $\Delta P$ is calculated from the microwave transmittance data for the same sample in Fig. 2(a) 17]. As shown in Fig. 2(c), the magnitude of $\left|V^{a} / \Delta P\right|$ monotonically increases with decreasing $T$ from $220 \mathrm{~K}$ to $40 \mathrm{~K}$ for $\mathrm{Py}|\mathrm{Cu}| \mathrm{SnBTS} 1$.

Figure 3 summarizes the $T$ dependence of the antisymmetric signals for all the samples. Here, the antisymmetric signal $\left|V^{a} / \Delta P\right|$ is normalized by the sample size; the produced electric field $E^{a} \equiv V^{a} / l_{\mathrm{Cu} \mid \mathrm{Py}}$ divided by the resonance absorption power per unit area $\Delta \tilde{P}$ is plotted in Fig. 3. As shown in Fig. 3, $\left|E^{a} / \Delta \tilde{P}\right|$ for the three samples is small and almost constant above $200 \mathrm{~K}$. The magnitudes of $\left|E^{a} / \Delta \tilde{P}\right|$ are similar for all the samples in the high $T$ range. This small constant signal at high temperatures may result from ferromagnetic transports in the Py layer [17]. With decreasing temperature below $150 \mathrm{~K}-200 \mathrm{~K},\left|E^{a} / \Delta \tilde{P}\right|$ begins to increase rapidly for the $\mathrm{Py}|\mathrm{Cu}| \mathrm{SnBTS}$ samples. This sharp enhancement of $\left|E^{a} / \Delta \tilde{P}\right|$ at low temperatures is clearly

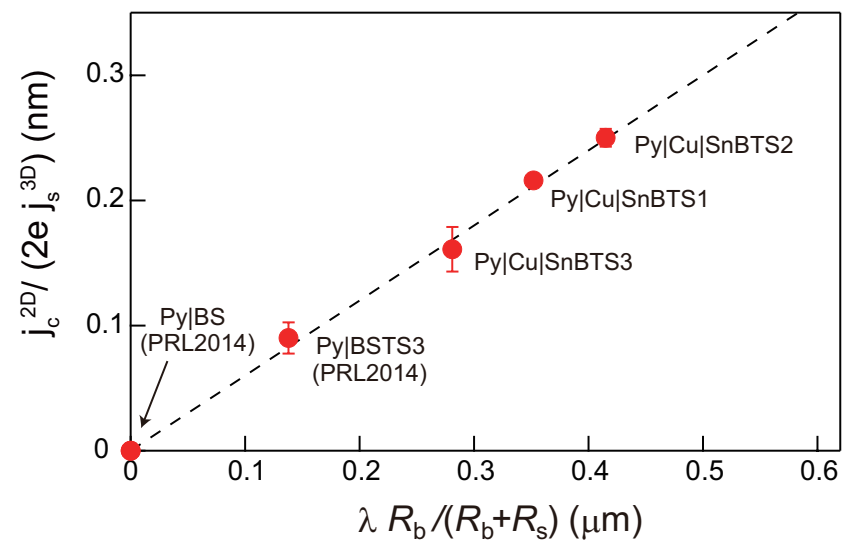

FIG. 4: (Color online.) The inverse Edelstein effect length, $j_{c}^{2 D} /\left(2 e j_{s}^{3 D}\right)$, as a function of $\lambda R_{s} /\left(R_{s}+R_{b}\right)$ for various samples. $j_{c}^{2 D}$ and $j_{s}^{3 D}$ indicate the electric current density generated on the TI surface and the spin current density injected from Py, respectively. Here, $e, \lambda, R_{s}$, and $R_{b}$ denote the elementary charge, the mean-free path on the surface, and the sheet resistances for the surface and bulk parts, respectively. The voltage-peak magnitude at the lowest temperature was used to calculate $j_{c}^{2 D}$. The experimental results for Py|BS and Py|BSTS3 reported in [17] are also plotted in this figure.

correlated with the resistivity increase at low temperatures, as shown by the dotted curve in Fig. 3. As the bulk resistivity increases at lower temperatures, the spin polarization accumulated near the TI surface increases and greater spin-electricity conversion signals may appear. The magnitude of $\left|E^{a} / \Delta \tilde{P}\right|$ at the lowest temperature is similar for all the samples, whereas the resistivity values are rather different among them [Fig. 1(b)].

In Fig. 4. we analyze the efficiency of the spinelectricity conversion in terms of the mean-free path on the surface $\lambda$ for each sample. The inverse Edelstein effect length 24 26] for the $\mathrm{Py}|\mathrm{Cu}| \mathrm{SnBTS}$ samples and also for $\mathrm{Py} \mid \mathrm{Bi}_{2} \mathrm{Se}_{3}(\mathrm{Py} \mid \mathrm{BS})$ and $\mathrm{Py} \mid \mathrm{Bi}_{1.5} \mathrm{Sb}_{0.5} \mathrm{Te}_{1.7} \mathrm{Se}_{1.3} 3$ (Py|BSTS3) reported in [17] (see also 27]) is calculated by dividing the surface electric current density $j_{c}^{2 D} \equiv$ $\left\{E^{a}(10 \mathrm{~K})-E^{a}(293 \mathrm{~K})\right\} / R_{t}$ by the spin current density $j_{s}^{3 D}$ [5], where the electric current originating from the ferromagnetic transports in the Py layer $\left(E^{a}(293 \mathrm{~K}) / R_{t}\right)$ is subtracted. The obtained $j_{c}^{2 D} /\left(2 e j_{s}^{3 D}\right)$ is plotted against $\lambda R_{b} /\left(R_{b}+R_{s}\right)$ for each sample in Fig. 4. Here, $R_{t}, R_{s}$, and $R_{b}$ are the total sheet resistance for the $\mathrm{Py} \mid \mathrm{Cu}$ layer and the TI surface in parallel, the surface sheet resistance, and the bulk sheet resistance, respectively. $\lambda$ is estimated from the reported Fermi wavenumber $k_{F}\left(5.9 \times 10^{-2} \AA^{-1}\right.$ for Sn-BTS 20, 23] and $1.0 \times 10^{-1}$ $\AA^{-1}$ for BSTS [28]) and $R_{s}$ estimated for each sample 29], and $R_{b}$ and $R_{s}$ are separated from each other by fits to the $\rho$ - $T$ curve using the three-dimensional variable range hopping formula $\left(\sim T^{-1 / 4}\right)$ in a low- $T$ range [19]. For the bulk-metallic TI samples (BS), $R_{b} /\left(R_{b}+R_{s}\right) \approx 0$, and the spin-electricity conversion signal is not observed [17] (Fig. [4). For the bulk-insulating TI samples, by con- 
trast, both $j_{c}^{2 D}$ and $R_{b} /\left(R_{b}+R_{s}\right)$ exhibit sizable values. As shown in Fig. 4, a clear linear relation is observed, which shows that the spin-electricity conversion efficiency is greater for TI samples with higher bulk resistivity and longer surface mean-free path. Experimental results for Py|BSTS1 and Py|BSTS2 in literature [17] are not shown in Fig. 4. since the antisymmetric parts of the voltage peaks are not discerned above $100 \mathrm{~K}$ due to very large Seebeck voltages [17.

The linear dependence of the inverse Edelstein effect length on mean free path on the surface is consistent with the theoretical prediction [24 26]. The relation between the generated electric current and the spin polarization $\langle\sigma\rangle$ is given by $j_{c}^{2 D} \approx 2 e v_{F}\langle\sigma\rangle\left[30\right.$, where $v_{F}$ is the Fermi velocity for the surface state. The spin polarization on the surface is produced by injection of the spin current $j_{s}^{3 D}$ from Py, following $\langle\sigma\rangle / \tau=j_{s}^{3 D} R_{b} /\left(R_{b}+R_{s}\right)(\tau$ being the scattering relaxation time for the surface) [17]. Hence, we obtain $j_{c}^{2 D} / j_{s}^{3 D} \approx 2 e \lambda R_{b} /\left(R_{b}+R_{s}\right)$. This relation well explains the experimental results in Fig. 4. It is interestingly noted that the slope of the linear fit in Fig. 4 corresponds to the spin injection efficiency $\eta$ 17]. The obtained value is $\eta \approx 6.0 \times 10^{-4}(0.06 \%)$, which is comparable to the values reported for bulk-insulating BSTS samples [17, 22].

In summary, we measured spin-electricity conversion induced by spin pumping into Sn-doped $\mathrm{Bi}_{2} \mathrm{Te}_{2} \mathrm{Se}$ at low temperatures. To prevent magnetic proximity effects from ferromagnetic layers, a thin $\mathrm{Cu}$ layer was inserted between Py and TI. At FMR in the Py, spin-electricity conversion voltage was observed and enhanced with decreasing temperature. The spin-electricity conversion efficiency at low temperatures is found to increase with increasing magnitudes of bulk resistivity and surface meanfree path, following a single scaling law. This result is consistent with theories on spin-electricity conversion on the topological surface state.

K.T.Y. acknowledges the support through the Leading Graduates Schools Program (Tohoku University "MD program") by the MEXT. Y.S. would like to acknowledge the support from the Motizuki Fund of Yukawa Memorial Foundation. This work was supported by JSPS (KAKENHI No. 25220708 and the Core-to-Core program "International research center for new-concept spintronics devices") and MEXT (Innovative Area gNano Spin Conversion Scienceh (No. 26103005)).
[1] S. Maekawa, S.O. Valenzuela, E. Saitoh, and T. Kimura, Spin Current, (Oxford University Press, Oxford, 2012).

[2] E. Saitoh, M. Ueda, H. Miyajima, and G. Tatara, Appl. Phys. Lett. 88, 182509 (2006).

[3] A. Azevedo, L.H.V. Leao, R.L. Rodriguez-Suarez, A.B. Oliveira, and S.M. Rezende, J. Appl. Phys. 97, 10C715 (2005).

[4] M.V. Costache, M. Sladkov, S.M. Watts, C.H. van der Wal, and B.J. van Wees, Phys. Rev. Lett. 97, 216603 (2006).

[5] K. Ando, S. Takahashi, J. Ieda, Y. Kajiwara, H. Nakayama, T. Yoshino, K. Harii, Y. Fujikawa, M. Matsuo, S. Maekawa and E. Saitoh, J. Appl. Phys. 109, 103913 (2011).

[6] O. Mosendz, J.E. Pearson, F.Y. Fradin, G.E.W. Bauer, S.D. Bader, and A. Hoffmann, Phys. Rev. Lett. 104, 046601 (2010).

[7] Y. Niimi, Y. Kawanishi, D.H. Wei, C. Deranlot, H.X. Yang, M. Chshiev, T. Valet, A. Fert, and Y. Otani, Phys. Rev. Lett. 109, 156602 (2012).

[8] K. Fujiwara, Y. Fukuma, J. Matsuno, H. Idzuchi, Y. Niimi, Y. Otani and H. Takagi, Nat. Commun. 4, 2893 (2013).

[9] A.R. Mellnik, J.S. Lee, A. Richardella, J.L. Grab, P.J. Mintun, M.H. Fischer, A. Vaezi, A. Manchon, E.-A. Kim, N. Samarth, and D.C. Ralph, Nature 511, 449 (2014).

[10] Y. Fan, P. Upadhyaya, X. Kou, M. Lang, S. Takei, Z. Wang, J. Tang, L. He, L.-T. Chang, M. Montazeri, G. Yu, W. Jiang, T. Nie, R.N. Schwartz, Y. Tserkovnyak, and K.L. Wang, Nature Mater. 13, 699-704 (2014).

[11] Y. Ando, J. Phys. Soc. Jpn. 82, 102001 (2013).

[12] M.Z. Hasan and C.L. Kane, Rev. Mod. Phys. 82, 30453067 (2010).

[13] X.-L. Qi, and S.-C. Zhang, Rev. Mod. Phys. 83, 1057-
1110 (2011).

[14] A.A. Baker, A.I. Figueroa, L.J. Collins-McIntyre, G. van der Laan, and T. Hesjedal, Sci. Rep. 5, 7907 (2015).

[15] P. Deorani, J. Son, K. Banerjee, N. Koirala, M. Brahlek, S. Oh, and H. Yang, Phys. Rev. B 90, 094403 (2014).

[16] M. Jamali, J.S. Lee, J.S. Jeong, F. Mahfouzi, Y. Lv, Z. Zhao, B.K. Nikolic, K.A. Mkhoyan, N. Samarth, and J.P. Wang, Nano Lett. 15, 7126-7132 (2015).

[17] Y. Shiomi, K. Nomura, Y. Kajiwara, K. Eto, M. Novak, K. Segawa, Y. Ando, and E. Saitoh, Phys. Rev. Lett. 113, 196601 (2014).

[18] A.A. Taskin, Z. Ren, S. Sasaki, K. Segawa, and Y. Ando, Phys. Rev. Lett. 107, 016801 (2011).

[19] Z. Ren, A.A. Taskin, S. Sasaki, K. Segawa, and Y. Ando, Phys. Rev. B 84, 165311 (2011).

[20] Z. Ren, A.A. Taskin, S. Sasaki, K. Segawa, and Y. Ando, Phys. Rev. B 85, 155301 (2012).

[21] S. Hong, V. Diep, S. Datta, and Y.P. Chen, Phys. Rev. B 86, 085131 (2012).

[22] Y. Ando, T. Hamasaki, T. Kurokawa, K. Ichiba, F. Yang, M. Novak, S. Sasaki, K. Segawa, Y. Ando, and M. Shiraishi, Nano Lett. 14, 6226-6230 (2014).

[23] S.K. Kushwaha, Q.D. Gibson, J. Xiong, I. Pletikosic, A.P. Weber, A.V. Fedorov, N.P. Ong, T. Valla, and R.J. Cava, J. Appl. Phys. 115, 143708 (2014).

[24] J.C. Rojas-Sánchez, L. Vila, G. Desfonds, S. Gambarelli, J.P. Attane, J.M. De Teresa, C. Magen and A. Fert, Nat. Commun. 4, 2944 (2013).

[25] J.C. Rojas-Sánchez, S. Oyarzun, Y. Fu, A. Marty, C. Vergnaud, S. Gambarelli, L. Vila, M. Jamet, Y. Ohtsubo, A. Taleb-Ibrahimi, P. Le Fevre, F. Bertran, N. Reyren, J.-M. George, and A. Fert, Phys. Rev. Lett. 116, 096602 (2016).

[26] K. Shen, G. Vignale, and R. Raimondi, Phys. Rev. Lett. 
112, 096601 (2014).

[27] The experimental results in [17] are re-analyzed for the plot in Fig. 4 For Py|BSTS3, the temperature dependence of $V^{a} / \Delta P$ is shown in Fig. S11 in Supplemental Material of [17].

[28] T. Arakane, T. Sato, S. Souma, K. Kosaka, K. Nakayama,
M. Komatsu, T. Takahashi, Zhi Ren, Kouji Segawa and Yoichi Ando, Nature Commun. 3, 636 (2011).

[29] D. Culcer, Physica (Amsterdam) 44E, 860 (2012).

[30] A.A. Burkov and D.G. Hawthorn, Phys. Rev. Lett. 105, 066802 (2010). 